\title{
Surface plasmon resonance detection of blood coagulation and platelet adhesion under venous and arterial shear conditions
}

\author{
Kenny Hansson, Knut Johansen, Jonas Wetterö, Goran Klenkar, Johan Benesch, \\ Ingemar Lundström, Tomas Lindahl and Pentti Tengvall
}

\section{Linköping University Post Print}

N.B.: When citing this work, cite the original article.

Original Publication:

Kenny Hansson, Knut Johansen, Jonas Wetterö, Goran Klenkar, Johan Benesch, Ingemar Lundström, Tomas Lindahl and Pentti Tengvall, Surface plasmon resonance detection of blood coagulation and platelet adhesion under venous and arterial shear conditions, 2007, Biosensors \&amp; bioelectronics, (23), 2, 261-268.

http://dx.doi.org/10.1016/j.bios.2007.04.009

Copyright: Elsevier

http://www.elsevier.com/

Postprint available at: Linköping University Electronic Press http://urn.kb.se/resolve?urn=urn:nbn:se:liu:diva-39210 


\title{
Surface plasmon resonance detection of blood coagulation and platelet adhesion under venous and arterial shear conditions
}

\author{
Kenny M. Hansson ${ }^{1}$, Knut Johansen ${ }^{2}$, Jonas Wetterö ${ }^{3}$, Goran Klenkar ${ }^{4}$, Johan Benesch ${ }^{5}$, \\ Ingemar Lundström ${ }^{4}$, Tomas L. Lindahl ${ }^{6}$, and Pentti Tengvall ${ }^{4}$
}

\footnotetext{
${ }^{I}$ At present: AstraZeneca R\&D Mölndal, SE-431 83 Mölndal, Sweden. During the work presented: Division of Applied Physics, Department of Physics, Chemistry and Biology, Linköping University, SE58183 Linköping, Sweden

${ }^{2}$ Scientific Engineering QED, Onkel Adamsgatan 9, SE-582 35 Linköping, Sweden

${ }^{3}$ Division of Rheumatology/AIR, Department of Molecular and Clinical Medicine, Linköping University, SE-581 85 Linköping, Sweden

${ }^{4}$ Division of Applied Physics, Department of Physics, Chemistry and Biology, Linköping University, SE58183 Linköping, Sweden

53B's Research Group - Biomaterials, Biodegradables and Biomimetics, Department of Polymer Engineering, University of Minho, Campus de Gualtar, 4710-057 Braga, Portugal ${ }^{6}$ Division of Clinical Chemistry, Department of Biomedicine and Surgery, Linköping University, SE-581 85 Linköping, Sweden.
}

\begin{abstract}
A surface plasmon resonance (SPR) based flow chamber device was designed for real time detection of blood coagulation and platelet adhesion in platelet rich plasma and whole blood. The system allowed the detection of surface interactions throughout the 6 $\mathrm{mm}$ length of the flow chamber. After deposition of thromboplastin onto a section of the
\end{abstract}


sensor surface near the inlet of the flow chamber, coagulation was detected downstream of this position corresponding to a SPR signal of 7 to $8 \mathrm{mRIU}$ ( 7 to $8 \mathrm{ng} / \mathrm{mm}^{2}$ ). A nonmodified control surface induced coagulation 3.5 times slower. Platelet adhesion to gold and fibrinogen coated surfaces in the magnitude of 1.25 and $1.66 \mathrm{mRIU}$ was also shown with platelets in buffer, respectively. SPR responses obtained with platelet rich plasma and whole blood on surfaces that were methylated or coated with von Willebrand factor, fibrinogen, or collagen, coincided well with platelet adhesion as observed with fluorescence microscopy in parallel experiments. The present SPR detection equipped flow chamber system is a promising tool for studies on coagulation events and blood cell adhesion under physiological flow conditions, and allows monitoring of short-range surface exerted processes in whole blood.

Keywords: surface plasmon resonance, flow chamber, platelet, coagulation, whole blood, blood plasma

* Corresponding author. E-mail address: kenny.m.hansson@astrazeneca.com 


\section{Introduction}

Flow induced shear is pivotal for the complex in vivo performance of the haemostatic system and affects largely, e.g. blood coagulation and platelet adhesion and activation. However, due to experimental constraints, most methods that study haemostasis lack controlled shear. There exist a few relevant flow chamber systems (Sakariassen et al., 1983, Badimon et al., 1987), yet the detection of molecular events and evaluation of results is often cumbersome, time consuming, and can not be performed in real time. This makes these flow chamber systems less useful although they more accurately describe the in vivo situation than static methods. The presently available real time flow chamber systems are predominantly based on fluorescence microscopy techniques and require

labelling of either blood cells or proteins (Tsuji et al., 1999, Moroi et al., 2000, Kuwahara et al., 2002). In the present work, real-time surface plasmon resonance technique was applied in a flow chamber. The set-up is demonstrated functional for studies on blood protein mediated coagulation, and platelet adhesion during relevant venous and arterial shear. 


\section{Materials and Methods}

\section{Blood, plasma and platelets}

Heparinised (5 IU/ml) and citrated blood plasma $(0.011 \mathrm{~mol} / \mathrm{l})$ were pooled from two apparently healthy donors at the Blood Transfusion Unit at Linköping University Hospital. The plasma was aliquoted and stored at $-80^{\circ} \mathrm{C}$ until use.

Before use, the plasma was thawed at $37^{\circ} \mathrm{C}$ and then temperature-equilibrated to $22^{\circ} \mathrm{C}$. In order to obtain citrated platelet free plasma (PFP), the plasma was filtered through a $0.22 \mu \mathrm{m}$ sterile filter (MILLEX-GV, Millipore, Bedford, MA, USA) at room temperature. Citrated $(0.011 \mathrm{~mol} / \mathrm{l})$ whole blood and platelet rich plasma (PRP) were collected by the same unit as described above. The platelet count was standardized to $200 \times 10^{9} / 1$ in PRP through the addition of citrated PFP.

Heparinised blood $(5 \mathrm{IU} / \mathrm{ml})$ was used for platelet preparation at room temperature as described in (Bengtsson and Grenegard, 1994). The platelets were suspended in KrebsRinger phosphate buffer, $\mathrm{pH} 7.3$ (KRG) and displayed no apparent activation due to the preparation procedure.

All blood donors had given their informed consent and the local ethics committee approved the blood collection protocol.

\section{Reagents}

Thromboplastin purified from rabbit brain was a kind gift from Global Hemostasis Institute MGR AB, Linköping, Sweden. The general rinsing buffer hepes buffered saline 
(HBS-N) pH 7.4 and the SPR gold sensor surfaces with $45 \mathrm{~nm}$ gold on glass were from GE Healthcare, Uppsala, Sweden.

Collagen S type I was from calf skin (Roche Diagnostics GmbH, Mannheim, Germany). The von Willebrand factor concentrate Haemate (Centeon, Stockholm, Sweden) also contains coagulation factor VIII and albumin. The $\mathrm{HS}\left(\mathrm{CH}_{2}\right)_{16} \mathrm{CH}_{3}$ reagent used for surface methylations, cysteamine and ethanolamine were purchased from Fluka (Basel, Switzerland). Fibrinogen was delivered by Chromogenix $\mathrm{AB}$ (Sweden) and $\mathrm{CaCl}_{2} \cdot 2 \mathrm{H}_{2} \mathrm{O}$ by Merck GmbH (Darmstadt, Germany). Trypsin and sucrose were from Sigma (St Louis, MO, USA). Sodium dodecyl sulphate (SDS) was from Bio-Rad Laboratories (Hercules, CA, USA).

\section{Surface preparations and adsorption protocols}

The sensor surfaces were cleaned in a 1:1:5 mixture of 30\% $\mathrm{H}_{2} \mathrm{O}_{2}$ (Merck), $25 \% \mathrm{NH}_{3}$ (Merck) and Milli-Q water at $80^{\circ} \mathrm{C}$ for five minutes, yielding clean hydrophilic surfaces with contact angles $<20^{\circ}$.

Gold surfaces with adsorbed thromboplastin were produced by placing a narrow band ( 2 $\mathrm{mm})$ of thromboplastin solution (10 $\mathrm{U} / \mathrm{ml}$ in HBS-N) perpendicular to the flow direction near the inlet part of the surface for 10 minutes. The modified area was then rinsed gently to avoid contamination of the unmodified areas. After mounting into the flow chamber, the surface was exposed to a continuous buffer flow to remove material not firmly attached to the surface.

Fibrinogen coated gold surfaces were obtained by incubation of clean hydrophilic gold surfaces in a fibrinogen solution, $1 \mathrm{~g} / 1$ in phosphate buffered saline (PBS) pH 7.4 for 30 minutes. 
Self assembled hydrophobic monolayers (SAM) on clean gold surfaces were prepared through chemisorption of $\mathrm{HS}\left(\mathrm{CH}_{2}\right)_{16} \mathrm{CH}_{3}$ in a $99.5 \%$ ethanol solution ( $2 \mathrm{mmol} / \mathrm{l}$ ) overnight (Lestelius et al., 1997).

Collagen coated gold surfaces were obtained through incubation of clean gold surfaces in cysteamine (10 mmo1/l) for 48 hours, followed by transition to glutaraldehyde in PBS $(6 \%$, $\mathrm{pH}$

8) during 50 minutes, to Collagen $\mathrm{S}(0.1 \mathrm{mg} / \mathrm{ml})$ for 48 hours and finally incubation in ethanolamine (1 mol/l) in PBS pH 8 during 2 hours.

Sensor surfaces coated with vWF were obtained by incubation of metylated gold surfaces in Haemate during 1 hour. All surfaces were rinsed in milli-Q water and dried in flowing $\mathrm{N}_{2}$ prior to use.

\section{Experimental}

\subsection{Optical system and flow chamber}

Surface concentration on the sensor was measured by an SPR set-up, which measures the resonance angle, i.e. the angle where minimum of reflected intensity occurs. A tungsten halogen light source (TS-428) and a monochromator (SpectraPro-300i) was used to obtain a monochromatic light of $633 \mathrm{~nm}$, both units from Acton Research Corporation, Acton, MA, USA. The light passed BK7 plano-cylindrical lenses (Melles Griot, USA) with refractive index $n_{0}=1.5168$. A dichroich sheet polarizer (Melles Griot) was placed in the light beam to obtain p-polarised light. The light was fan shape focused onto the sensor surface through a plano-cylindrical prism. The optical contact between the prism and the 
sensor surface was obtained by use of index matching fluid (Cargille, USA) with refractive index $n=1.5$. The reflected, divergent fan-shaped beam was collected by a CCD camera (CV-M50, JAI, Denmark), covering approximately 6 degrees in 640 pixels. An outline of the SPR instrumentation with flow chamber is shown in Figure 1. An in-house produced flow chamber made of polymethylmethacrylate (PMMA) was placed under the sensor surface with dimensions selected to result in a cross section area of $2 \mathrm{~mm}^{2}$ and a laminar flow profile inside the chamber. The sample solutions entered the flow chamber at a low angle visavi the surface and left it in a direction normal to the surface. The silicon tubing (Alitea $\mathrm{AB}$, Sweden) connecting the flow chamber with the sample or buffer supply and the waste had a cross section area of $2 \mathrm{~mm}^{2}$. The peristaltic pump was placed downstream to avoid interference with the sample prior to passage through the flow chamber.

\subsection{Data collection and analysis}

Digital images were acquired at $1 \mathrm{~Hz}$ with a 10 bit ADC frame grabber (PCI-1409, National Instruments, USA). The position of the resonance angles was obtained by an interpolation procedure. The position of the resonance dip was normalized to refractive index units (RIU) by linear regression from recorded pixel values of four sucrose solutions $(0,4,8$, and $12 \%(\mathrm{w} / \mathrm{w}))$ with known refractive indices. A pixel displacement of 1 unit was calculated to equal approximately $5 \cdot 10^{-5}$ RIU. One (1) mRIU corresponds to about $1 \mathrm{ng} / \mathrm{mm}^{2}$ of organic material on the surface. The equivalent refractive index will be used as an indication of the amount of organic material adsorbed. 
The noise level for the whole system was measured with a gold surface and a flowing buffer for at least 10 seconds, leading to a standard deviation of $0.0058 \mathrm{mRIU}$. Moreover, the resolution was at least a magnitude better, i.e. $0.0005 \mathrm{mRIU}$ based on the detector resolution (640 pixels) and the resolution of the analogue to digital converter (10-bits) (Johansen et al., 2000).

\subsection{Thromboplastin induced coagulation}

The partly thromboplastin-coated surfaces were mounted in the flow chamber. A pulse with HBS-N buffer was passed through the chamber for $10 \mathrm{~s}$, followed by a 2 minutes flow of heparinised plasma to hinder non-specific activation at the uncoated parts and finally approximately 15 minutes flow of recalcified citrated plasma. The citrated plasma was recalcified by addition of $25 \mu \mathrm{CaCl}_{2}(0.5 \mathrm{~mol} / \mathrm{l})$ per $1 \mathrm{ml}$ of citrated plasma immediately before injection into the flow chamber. The shear rate during the experiment was $100 \mathrm{~s}^{-1}$ corresponding to a venous shear rate (Hathcock, 2006). Control experiments with clean hydrophilic gold surfaces were performed in the same manner as described above. The experiments were performed in duplicate.

\subsection{Blood cell adhesion}

Platelet adhesion onto a clean hydrophilic gold surface was studied by first flowing HBS$\mathrm{N}$ for 10 seconds through the flow chamber, followed by platelets in buffer $\left(2.5 \times 10^{11} / 1\right)$ for 60 seconds and a final HBS-N pulse for 60 seconds.

Platelet adhesion to fibrinogen precoated gold surfaces was started with a HBS-N pulse

for 10 seconds, followed by flow of platelets in buffer $\left(2.5 \times 10^{11} / 1\right)$ during 5 minutes, and 
finally a HBS-N pulse during 60 seconds. The experiments were performed in duplicate and the shear rate was $700 \mathrm{~s}^{-1}$ corresponding to a low arterial shear rate (Hathcock, 2006). Platelet adhesion experiments with recalcified citrated PRP were performed on clean hydrophilic gold and methylated (heptadecanthiol) gold, collagen, and vWF at shear rates $50 \mathrm{~s}^{-1}$ (venous) and $1600 \mathrm{~s}^{-1}$ (arterial) (Goldsmith and Turitto, 1986, Hathcock, 2006), respectively. The clean gold and the methylated gold surfaces were control surfaces for the evaluation of the collagen and vWF coated surfaces. The experiment started with a 2 minutes flow of HBS-N. Recalcified citrated PFP was then perfused for 5 minutes followed by another 2 minutes of HBS-N to achieve a plasma protein saturated surface. Finally recalcified citrated PRP was perfused for 5 minutes followed by a period of 2 minutes with HBS-N.

The recalcification of PRP was made immediately before the start of each experiment by addition of $25 \mu \mathrm{CaCl}_{2}(0.5 \mathrm{~mol} / \mathrm{l})$ per $1 \mathrm{ml}$ of citrated plasma.

Experiments with adhering blood cells from recalcified citrated whole blood were performed with collagen and vWF coated surfaces at shear rates $50 \mathrm{~s}^{-1}$ (venous) and 1600 $\mathrm{s}^{-1}$ (arterial). The control surfaces were of the same type as for the PRP experiment. The experiment was started with a 2 minutes flow of HBS-N buffer. Subsequently recalcified citrated whole blood was perfused for 5 minutes followed by another 2 minutes flow of HBS-N.

The recalcification was performed immediately before the start of each experiment by addition of $17 \mu \mathrm{CaCl}_{2}(0.5 \mathrm{~mol} / \mathrm{l})$ per $1 \mathrm{ml}$ of citrated blood. After an individual experiment, the flow system was cleaned by flowing $0.3 \%(\mathrm{w} / \mathrm{w})$ SDS and trypsin $(1 \mathrm{~g} / \mathrm{l})$ in HBS-N buffer through the system for 30 minutes. 
Fluorescence microscopy was used to visualize the filamentous actin cytoskeleton and morphology of cells that adhered to the modified surfaces from platelet rich plasma and whole blood. The surfaces were gently washed in PBS placed in ice-cold 4\% paraformaldehyde in PBS during 40 minutes, and rinsed in ice-cold PBS. Then $150 \mu$ of a solution containing $100 \mu 1$ bodipy ${ }^{\circledR}$ phallacidin $(0.6 \mathrm{mg} / \mathrm{l}$, Molecular Probes Invitrogen, Eugene, OR, USA) and $200 \mu 1$ lysophosphatidylcholine (100 mg/l, Sigma, St. Louis, MO, USA) in $1.7 \mathrm{ml}$ PBS was placed on each surface and incubated for 40 minutes. The surfaces were rinsed in PBS and mounted using a fluorescence-mounting medium (Dako Cytomation AS, Glostrup, Denmark). The stained samples were excited and documented using an Axioscope equipped with a ZVS-47E digital camera (Carl Zeiss, Jena, Germany). The magnification was 630x or 1000x original magnification. Digital images were generated at a constant gain using Easy Image Measurement 2000 software (version 2.3, Bergströms Instrument AB, Solna, Sweden). 


\section{Results}

\section{Thromboplastin induced coagulation}

In order to study the activation of coagulation downstream a surface modification, one part of the surface facing the inlet was coated with thromboplastin in a $2 \mathrm{~mm}$ wide band perpendicular to the flow direction. See Supplementary data 1 . The modified surfaces were after stabilisation in flowing buffer exposed to flowing heparinised plasma to saturate them with proteins. This was done to differentiate the coagulation response obtained by interaction with the thromboplastin at the inlet, from the plasma protein adsorption itself when clottable recalcified plasma later was introduced. The plasma protein adsorption process was displayed as an immediate increase in signal downstream the inlet of between 12 and 14 mRIU (Table 1 and Figure 2), and with the largest increase (mass deposition) at the non-modified surface area between lines 3-7 (Table 1). When clottable recalcified citrated plasma was flown over the surface an additional increase in response was observed after $250 \mathrm{~s}$ total runtime with the highest signal increase downstream the thromboplastin coated inlet region (lines 3 to 7 in Table 1 and Figure 2). This coagulation specific surface response corresponded to a signal rise of about 7-8 mRIU. The control surface, i.e. an uncoated clean gold surface (Figure 2) showed a similar response due to plasma protein adsorption from heparinised plasma and the coagulation specific response in recalcified citrated plasma started slowly after approximately $860 \mathrm{~s}$ and was comparatively small. 


\section{Blood cell adhesion experiments}

To demonstrate that the adhesion of platelets could be monitored by the use of SPR flow equipment, platelets in buffer were flown over a clean gold surface. The results showed adhesion of mass, most probably platelets, onto the gold surface, corresponding to 1.28 $\mathrm{mRIU}\left(\sim 1.28 \mathrm{ng} / \mathrm{mm}^{2}\right)$ compared to baseline. To further monitor platelet adhesion the experiment was repeated with a fibrinogen coated sensor surface. The result showed adhesion of platelets on this surface, yielding an $1.66 \mathrm{mRIU}\left(\sim 1.66 \mathrm{ng} / \mathrm{mm}^{2}\right)$ increase compared to baseline.

To show that platelet adhesion monitoring also is possible in full physiological media, i.e. not only in neat buffer, an experimental series was performed in recalcified citrated PRP and another series with recalcified citrated whole blood at shear rates corresponding to venous $\left(50 \mathrm{~s}^{-1}\right)$ and arterial $\left(1600 \mathrm{~s}^{-1}\right)$ shear rates.

The SPR response due to PRP exposure, after subtraction of a signal corresponding to the PFP response, was most pronounced at the low shear rate for all types of surface coatings (Table 2). These results did not completely correspond to fluorescence micrographs from the same surfaces after fixing and staining of F-actin. Only clean gold at low shear and collagen coatings at high shear showed a substantial platelet surface coverage (Table 2 and Figure 3a). See also Supplementary data 2.

When whole blood was analysed on the same type of surfaces and at the same shear rates SPR responses were most pronounced on clean gold at low shear rate, followed by methylated surfaces at low shear rate, and vWF coated surfaces at low shear rate (Table 2). These results were confirmed by fluorescence microscopy (Table 2, Figure 3b). See also Supplementary data 2. 


\section{Discussion}

The flow induced shear is a key element of haemostasis which largely governs the platelet and coagulation activation and eventually the formation of a thrombus (Hathcock, 2006). The present work enables a real time measurement of haemostatic processes such as coagulation and platelet adhesion during controlled shear rates that correspond to typical venous and arterial shear rates.

The coagulation process in blood plasma and whole blood was previously detected with SPR during static conditions (Hansson et al., 1999, Hansson et al., 2002a, Hansson et al., 2002b, Vikinge et al., 2000). The results with thromboplastin-coated surfaces in the present work show that it is possible to detect blood plasma coagulation during dynamic conditions by the use of SPR as well. The coagulation did not occur in the area where thromboplastin was adsorbed onto the sensor surface but was detected downstream of this position. This is an important observation since most vascular endothelial damages in vivo results in a coagulum that is formed downstream the damaged part of the vessel wall and build up to cover the exposed subendothelium. The temporal coagulation response was about 3.5 fold faster for the thromboplastin coated surface when compared with a clean gold surface as reference.

Platelet adhesion and activation is a key event in the formation of thrombi in blood vessels. To demonstrate the usefulness of the SPR detection during platelet adhesion, isolated platelets were perfused in buffer through the flow chamber over a clean gold surface and a gold surface precoated with fibrinogen, respectively. In both cases an increase in SPR signal could be deduced to adhered platelets. The observed increase in signal was more pronounced on fibrinogen than clean gold, indicating a faster or firmer 
adhesion of platelets, although platelets are known to adhere to both gold (Wettero et al., 2003) and to fibrinogen via the GP IIb/IIIa receptors. In case of clean gold, a protein film mainly consisting of fibrinogen is formed on the surface upon exposure to blood or plasma fluids. Platelets then adhere to this protein film. In the present experiment the protein content was highly diluted since platelets were suspended in buffer and not plasma. Hence, it seems reasonable that the fibrinogen coated surface with a preformed and dense layer of fibrinogen present a more accessible adherence ground than the bare gold surface where a variety of proteins in low concentration, both adhesive and nonadhesive, become adsorbed prior to cell binding. It is thus clear that platelet adhesion can be monitored with SPR in buffer environment, but the most relevant media are platelet rich plasma or whole blood. To test these fluids, well-known pro-adhesive surfaces for platelets, i.e. vWF and collagen coated surfaces, were prepared.

In vivo, shear affected surface bound vWF is the binding site for platelet glycoprotein receptor Ib $\alpha(\mathrm{GPIb} \alpha)$ that mediates the initial adhesion of platelets at lesions in the blood vessel (Jung and Moroi, 2000). Surface exposed type I collagen is important for a firm platelet adhesion and activation via platelet glycoprotein receptor VI (GPVI) (Jung and Moroi, 2000). The also included reference surfaces clean hydrophilic and methylated gold initially interact with proteins and secondarily with blood cells. Both surfaces are known to adsorb fibrinogen, amongst many other plasma proteins and the methylated hydrophobic surface also adsorb vWF firmly (Siedlecki et al., 1996). Cross-comparison between the different types of surface coatings is not possible since it is difficult to estimate how close to the surface cells actually bind and how spread they 
are in relation to the SPR sensing depth and thickness of surface coating. Therefore, only comparisons between the same type of surfaces at low and high shear rate are discussed. One general observation from this study is a higher SPR signal during venous than arterial shear rates except for the collagen surface. This phenomenon is most probably explained by the fact that at higher shear the majority of loosely attached proteins and platelets become ripped off the surface. The clean gold and the methylated surfaces, known to bind fibrinogen, were extensively covered with platelets during the venous (low) but not with arterial (high) shear rates. This has been shown to be dependent on platelet GPIIb/IIIa-binding to adsorbed fibrinogen during low shear rates and the binding has been demonstrated to be markedly decreased at high shear rates (Zaidi et al., 1996). Similar findings has been reported for vWF, e.g. platelets do not bind firmly to vWF via GPIb $\alpha$ at arterial shear rates (Savage et al., 1996) and this might explain the low number of platelets that bound to this surface. Platelets need collagen to form initial loose attachment to collagen-captured vWF, and adhere later more firmly via GPVI to collagen itself, especially during high shear (Goto et al., 2002, Lecut et al., 2004). Higher shear rates activates platelets more easily and thereby expose more binding sites via exposed GPVI and thus gives a firmer adhesion. The present findings are in agreement with this since the current pattern for the collagen coated surfaces was a higher SPR signal during arterial than venous shear, accompanied by a higher platelet count (aggregates) in fluorescence microscopy.

The higher SPR response during arterial shear for the collagen surface may also reflect the morphological changes, i.e. spreading due to repolymerisation of the cytoskeleton, and not only firmer attachment of the platelets due to more extensive activation compared 
to venous shear. It was recently demonstrated that it is possible to resolve not only the cell adhesion area but also morphological changes in cells near the surface by the use of SPR (Yanase et al., 2006).

A general and expected observation in the present work is that surface exposure to whole blood results in higher SPR- and fluorescence microscopy signals than the corresponding PRP exposure. This is most probably due to differences in total blood cell content and other donor variations, due to the fact that the PRP and whole blood were from different donors and the PRP is by definition subjected to more extensive processing activities, such as centrifugation, which could affect platelet function. The haemodynamic process axial migration (Goldsmith, 1961) that results in increased density (2-3 times) of platelets near surfaces of a tubing while erythrocytes pass in the lumen of the tubing (Hathcock, 2006) may also partly explain the higher surface platelet numbers in the whole blood experiments than in PRP dito where there are no erythrocytes present. The differences due to donor variations, processing and haemodynamic performance may also account for the better correlation of SPR signal and surface coverage after whole blood exposure than after PRP exposure when comparing pair-wise for venous and arterial shear rates for all types of surfaces.

In conclusion, the SPR based flow chamber is applicable for the detection of coagulation phenomena and platelet/blood cell adhesion under physiological shear conditions. The potential of the flow chamber device depends on the specific application with highest applicability in biomaterials research to study surface protein/cell interactions in blood or blood derived media. In haemostatic studies it is better suited for studies on acute events during thrombus formation. This is because of the limited probe depth (approximately 
$100 \mathrm{~nm}$ ), that is insufficient to reveal the formation of typically mixed multilayers of cells and proteins that are formed as the result of extensive and prolonged coagulation activation. 


\section{Acknowledgement}

The Swedish Research Council (VR), Swedish Governmental Agency for Innovation Systems (VINNOVA), and the Swedish Society for Medical Research (SSMF) are gratefully acknowledged for financial support. Tomas Lindahl and Pentti Tengvall were supported by the strategic research area "Materials in Medicine" which is sponsored by County Council of Östergötland and the University of Linköping, Lindahl also by the Swedish Research Council project K2004-71X-15060-01A. We also want to thank Drs. Trine Platou Vikinge and Per-Erik Fägerman for their excellent technical support. 


\section{References}

BADIMON, L., TURITTO, V., ROSEMARK, J. A., BADIMON, J. J. \& FUSTER, V. (1987).[erratum appears in J Lab Clin Med 1988 Jan;111(1):5]. Journal of Laboratory \& Clinical Medicine, 110, 706-718.

BENGTSSON, T. \& GRENEGARD, M. (1994) European Journal of Cell Biology, 63, 345-349.

GOLDSMITH, H. L. (1961) Nature, 190, 1095-1096.

GOLDSMITH, H. L. \& TURITTO, V. T. (1986) Thromb Haemost, 55, 415-435.

GOTO, S., TAMURA, N., HANDA, S., ARAI, M., KODAMA, K. \& TAKAYAMA, H. (2002) Circulation, 106, 266-272.

HANSSON, K. M., TENGVALL, P., LUNDSTRÖM, I., RÅNBY, M. \& LINDAHL, T. L. (2002a) Biosensors and Bioelectronics, 17, 761-771.

HANSSON, K. M., TENGVALL, P., LUNDSTRÖM, I., RÅNBY, M. \& LINDAHL, T. L. (2002b) Biosensors and Bioelectronics, 17, 747-759.

HANSSON, K. M., VIKINGE, T. P., RÅNBY, M., TENGVALL, P., LUNDSTRÖM, I., JOHANSEN, K. \& LINDAHL, T. L. (1999) Biosensors and Bioelectronics, 14, $671-682$.

HATHCOCK, J. J. (2006) Arteriosclerosis, Thrombosis \& Vascular Biology, 26, 17291737.

JOHANSEN, K., STALBERG, R., LUNDSTROM, I. \& LIEDBERG, B. (2000) Measurement Science and Technology, 11, 1630-1638.

JUNG, S. M. \& MOROI, M. (2000) Trends in Cardiovascular Medicine, 10, 285-292. 
KUWAHARA, M., SUGIMOTO, M., TSUJI, S., MATSUI, H., MIZUNO, T., MIYATA, S. \& YOSHIOKA, A. (2002) Platelet shape changes and adhesion under high shear flow. Arteriosclerosis, Thrombosis \& Vascular Biology, 22, 329-34.

LECUT, C., SCHOOLMEESTER, A., KUIJPERS, M. J., BROERS, J. L., VAN ZANDVOORT, M. A., VANHOORELBEKE, K., DECKMYN, H., JANDROTPERRUS, M. \& HEEMSKERK, J. W. (2004) Arteriosclerosis, Thrombosis \& Vascular Biology, 24, 1727-33.

LESTELIUS, M., LIEDBERG, B. \& TENGVALL, P. (1997) LANGMUIR, 13, 59005908.

MOROI, M., ONITSUKA, I., IMAIZUMI, T. \& JUNG, S. M. (2000) Thrombosis \& Haemostasis, 83, 769-776.

SAKARIASSEN, K. S., AARTS, P. A., DE GROOT, P. G., HOUDIJK, W. P. \& SIXMA, J. J. (1983) J Lab Clin Med, 102, 522-535.

SAVAGE, B., SALDIVAR, E. \& RUGGERI, Z. M. (1996) Cell, 84, 289-297.

SIEDLECKI, C. A., LESTINI, B. J., KOTTKE-MARCHANT, K., EPPELL, S. J., WILSON, D. L. \& MARCHANT, R. E. (1996) Blood, 88, 2939-2950.

TSUJI, S., SUGIMOTO, M., MIYATA, S., KUWAHARA, M., KINOSHITA, S. \& YOSHIOKA, A. (1999) Blood, 94, 968-75.

WETTERO, J., ASKENDAL, A., TENGVALL, P. \& BENGTSSON, T. (2003) Journal of Biomedical Materials Research, 66A, 162-175.

VIKINGE, T. P., HANSSON, K. M., BENESCH, J., JOHANSEN, K., RÅNBY, M., LINDAHL, T. L., LIEDBERG, B., LUNDSTRÖM, I. \& TENGVALL, P. (2000) Journal of Biomedical Optics, 5, 51-55. 
YANASE, Y., SUZUKI, H., TSUSUI, T., HIRAGUN, T., KAMEYOSHI, Y. \& HIDE, M. (2006) Biosensors and Bioelectronics, 22, 1081-1086.

ZAIDI, T. N., MCINTIRE, L. V., FARRELL, D. H. \& THIAGARAJAN, P. (1996)

Blood, 88, 2967-2972. 


\section{Tables}

Table 1: SPR responses at different positions along the sensor surface in experiment with thromboplastin adsorption onto gold in a narrow band near the inlet of the flow chamber (lines 1-2), after perfusion with heparinised plasma, and a sequential pulse with recalcified citrated plasma, respectively. ( $1 \mathrm{mRIU}$ is estimated to correspond to approximately $1 \mathrm{ng} / \mathrm{mm}^{2}$ ) The values in the first column is to a large extent due to refractive index changes in the liquid outside the surface. The difference in $\Delta \mathrm{SPR}$ between the different positions is, however, significant

\begin{tabular}{ccc}
\hline Position on & $\Delta$ SPR response & $\Delta$ SPR signal \\
sensor surface & (Heparinised & (Citrated plasma - \\
(1 closest to & plasma - buffer & heparinised plasma \\
inlet) & response) (mRIU) & response) (mRIU) \\
\hline Line 1 & 12.455 & 3.385 \\
Line 2 & 14.838 & 4.043 \\
Line 3 & 14.047 & 6.240 \\
Line 4 & 14.372 & 7.077 \\
Line 5 & 14.424 & 7.630 \\
Line 6 & 14.438 & 7.868 \\
Line 7 & 14.608 & 8.113 \\
\hline
\end{tabular}


Table 2: Mean changes in SPR responses over 9 measuring lines and surface coverage determined with fluorescence microscopy. The fluorescence microscopy pictures were obtained after fixing and staining the same sensor surfaces that were analysed with SPR. Pulses with recalcified PFP, PRP, and whole blood were passed over pure gold, methylated gold, collagen coated gold, and von Willebrand factor (vWF) coated gold with shear rates corresponding to venous and arterial shear rates $\left(50 \mathrm{~s}^{-1}\right.$ and $1600 \mathrm{~s}^{-1}$, respectively). ( $1 \mathrm{mRIU}$ is approximately $1 \mathrm{ng} / \mathrm{mm}^{2}$ ) 


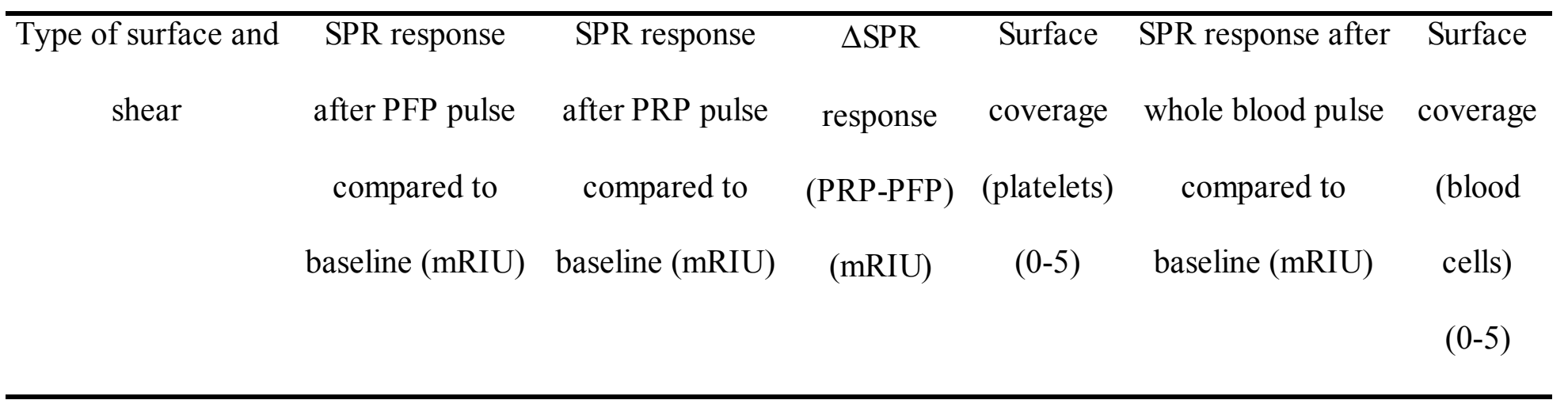

Hydrophilic gold,

venous shear

4.666

5.070

0.404

2

8.216

5

Hydrophilic gold,

arterial shear

4.492

4.066

$-0.426$

4.075

1

Methyl coating,

venous shear

2.664

2.536

$-0.128$

0

8.092

4

Methyl coating,

arterial shear

1.901

1.627

$-0.274$

1

5.238

0

vWF coating,

venous shear

1.215

1.568

0.353

6.355

5

vWF coating,

arterial shear

0.970

1.164

0.194

4.439

1

Collagen coating, venous shear

1.072

1.674

0.602

1

1.661

2

Collagen coating, arterial shear

2 


\section{Captions to figures:}

Figure 1: A schematic drawing showing the optical system and flowcell used for the SPR flow chamber measurements.

Figure 2: SPR response at line 5 upon flow (shear rate of $100 \mathrm{~s}^{-1}$ ) of recalcified citrated plasma (PFP) over thromboplastin adsorbed to gold (black line), and clean gold (grey line). First there was a pulse of buffer followed by heparinised plasma to saturate protein binding and finally recalcified plasma was introduced into the flow chamber. The introduction of different fluids into the flow chamber are indicated with arrows. Note that in this and following SPR results some of the rapid changes are due to refractive index changes in the fluid passing over the test surface and not entirely dependent on adsorbed mass.

Figure 3: Fluorescence microscopy images showing adhered blood cells after flow experiments with a) recalcified citrated PRP and b) recalcified citrated whole blood over clean gold, methylated gold, collagen coated gold, and von Willebrand factor coated sensor surfaces during venous (low, $50 \mathrm{~s}^{-1}$ ) and arterial (high, $1600 \mathrm{~s}^{-1}$ ) shear rates, respectively. Cells were fixed in paraformaldehyde and stained for filamentous actin. Bar length: $10 \mu \mathrm{m}$. 


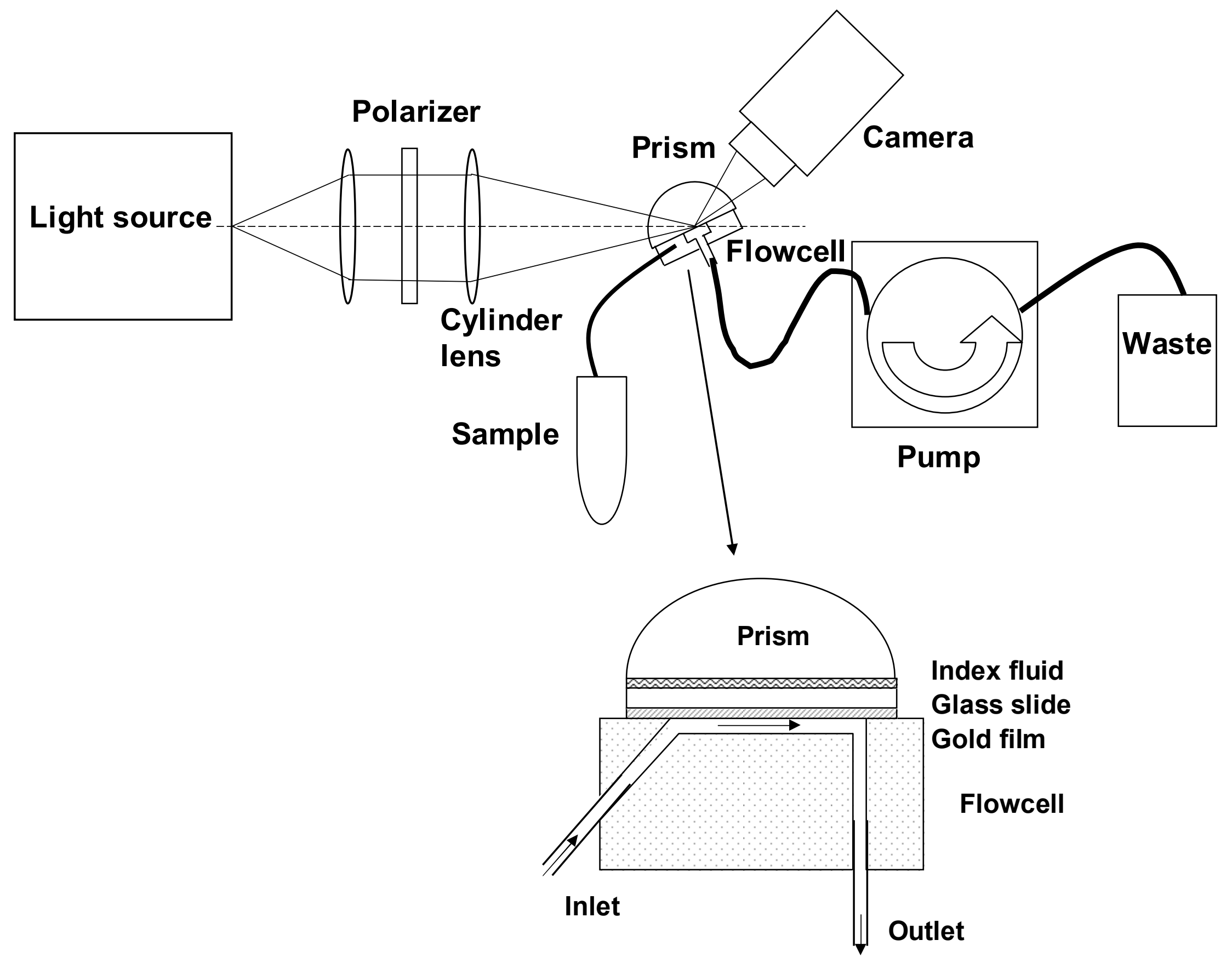




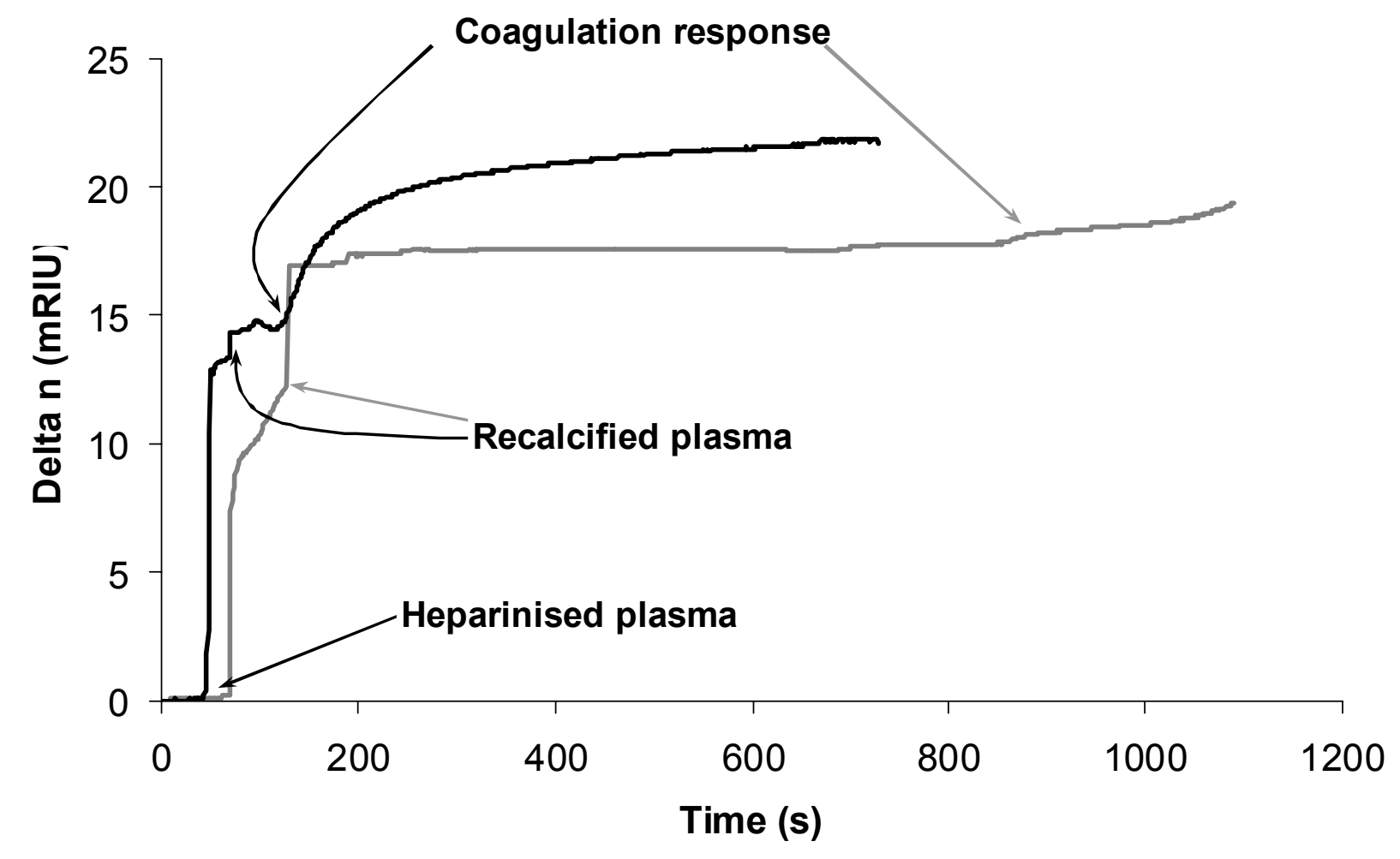


Figure $3 a$
Click here to download high resolution image

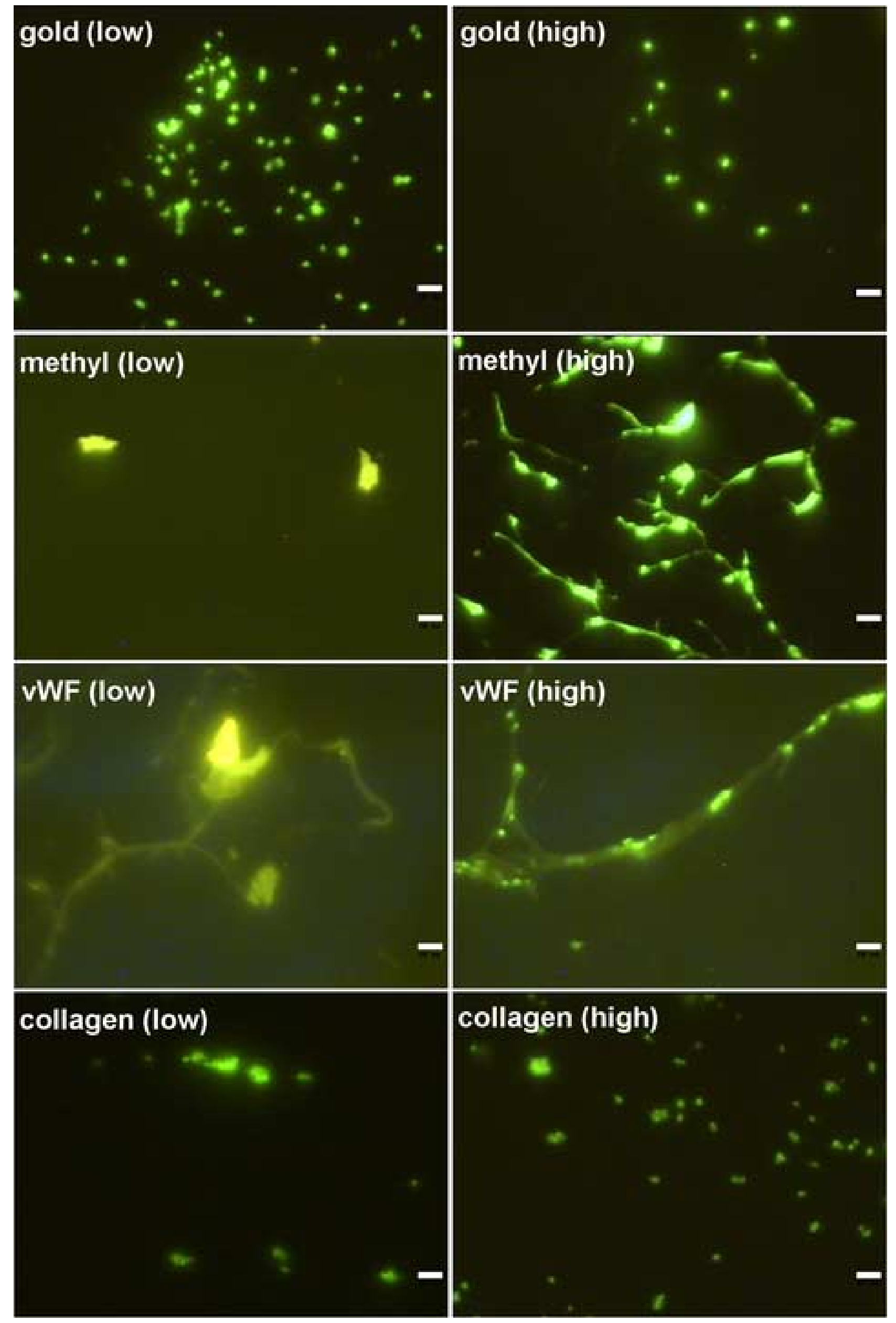


Figure 3b

Click here to download high resolution image

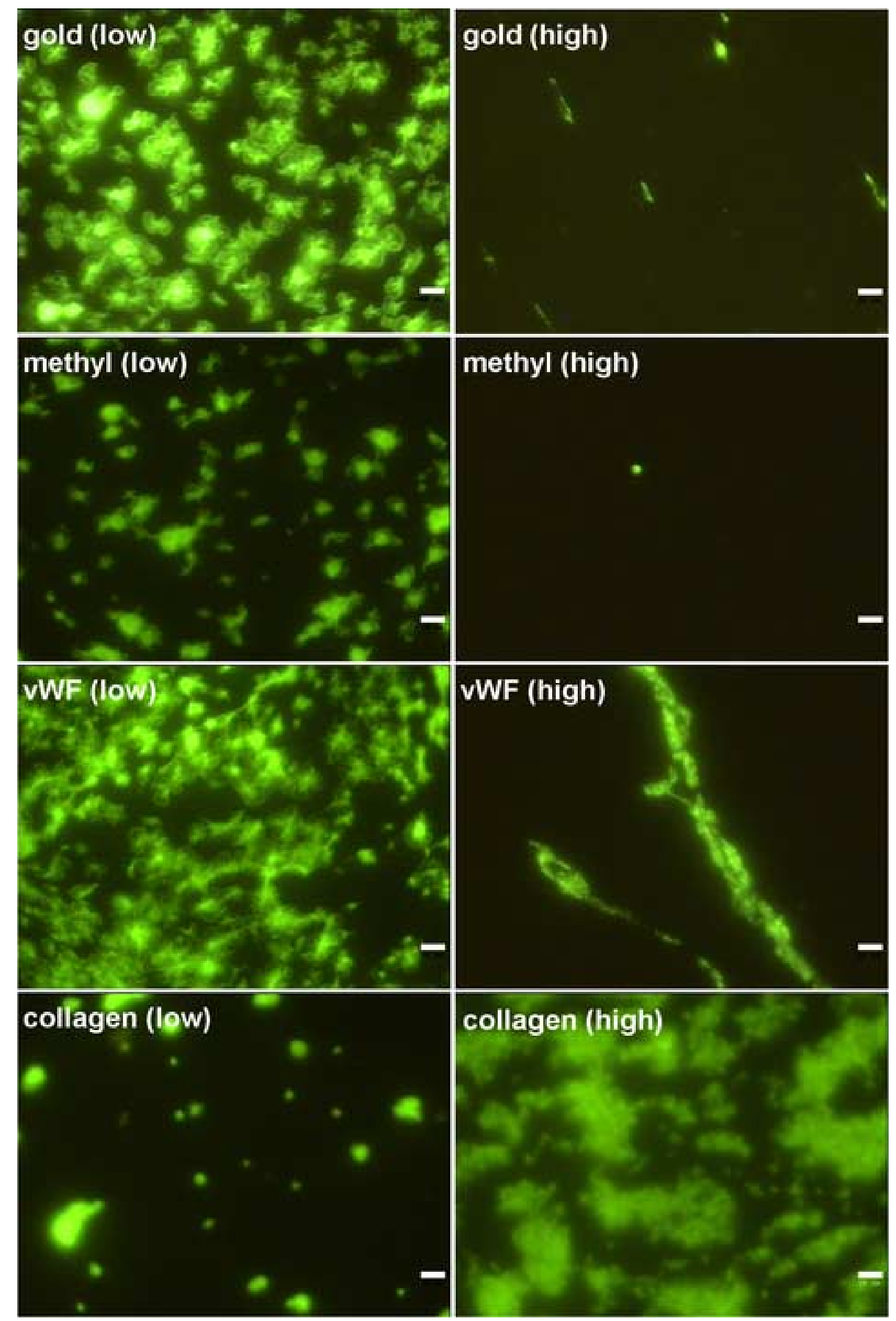

-

methyl (low)

methyl (high)

collagen (high) 
Supplementary data

\section{Supplement 1}

a)

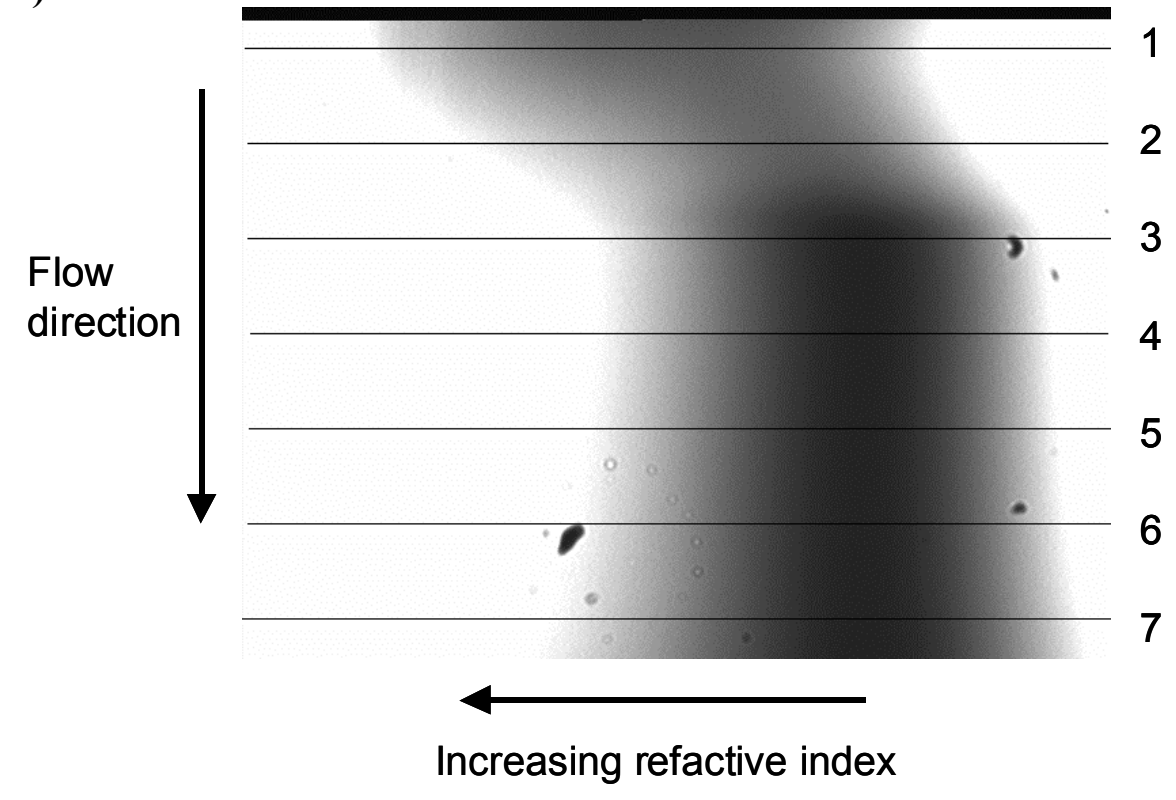

b)

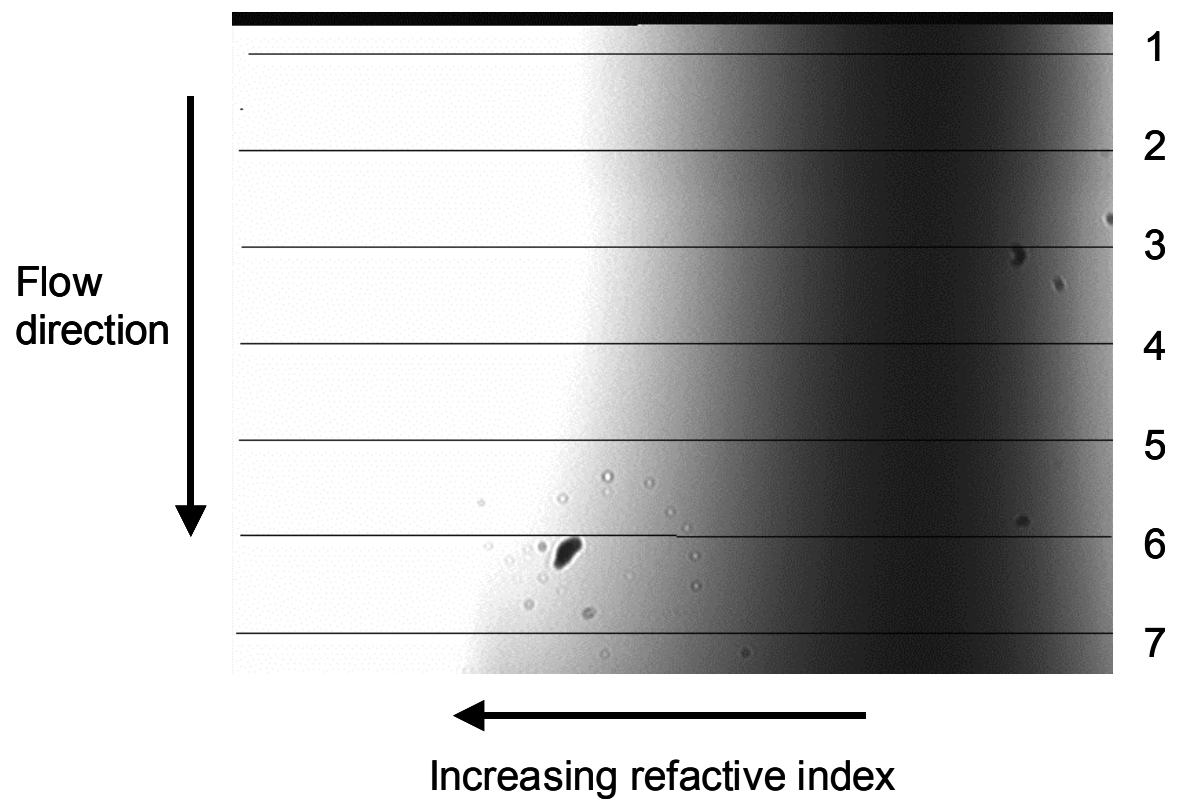

Supplement 1: Digital images showing the surface plasmon signal in RIU in terms of minimum in light reflection when a) HBS-N buffer was perfused over a partly thromboplastin-modified gold surface (lines 1-3) and b) HBS-N buffer was perfused over 
a clean gold surface. The lines in which measurements are performed are indicated to the right as well as the flow direction and direction of increasing refractive index. The dark area in the images is the area where the light reflection represents a minimum due to the surface plasmon resonance. A displacement of the dark areas to the left implies an increased refractive index due to mass deposition on the surface which in a) shows the adsorbed thromboplastin located between the indicated measuring lines 1 and 3, and very little, or no, deposited thromboplastin below line 3 and in b) shows that there is no deposition on the control surface. 


\section{Supplement 2}

a)

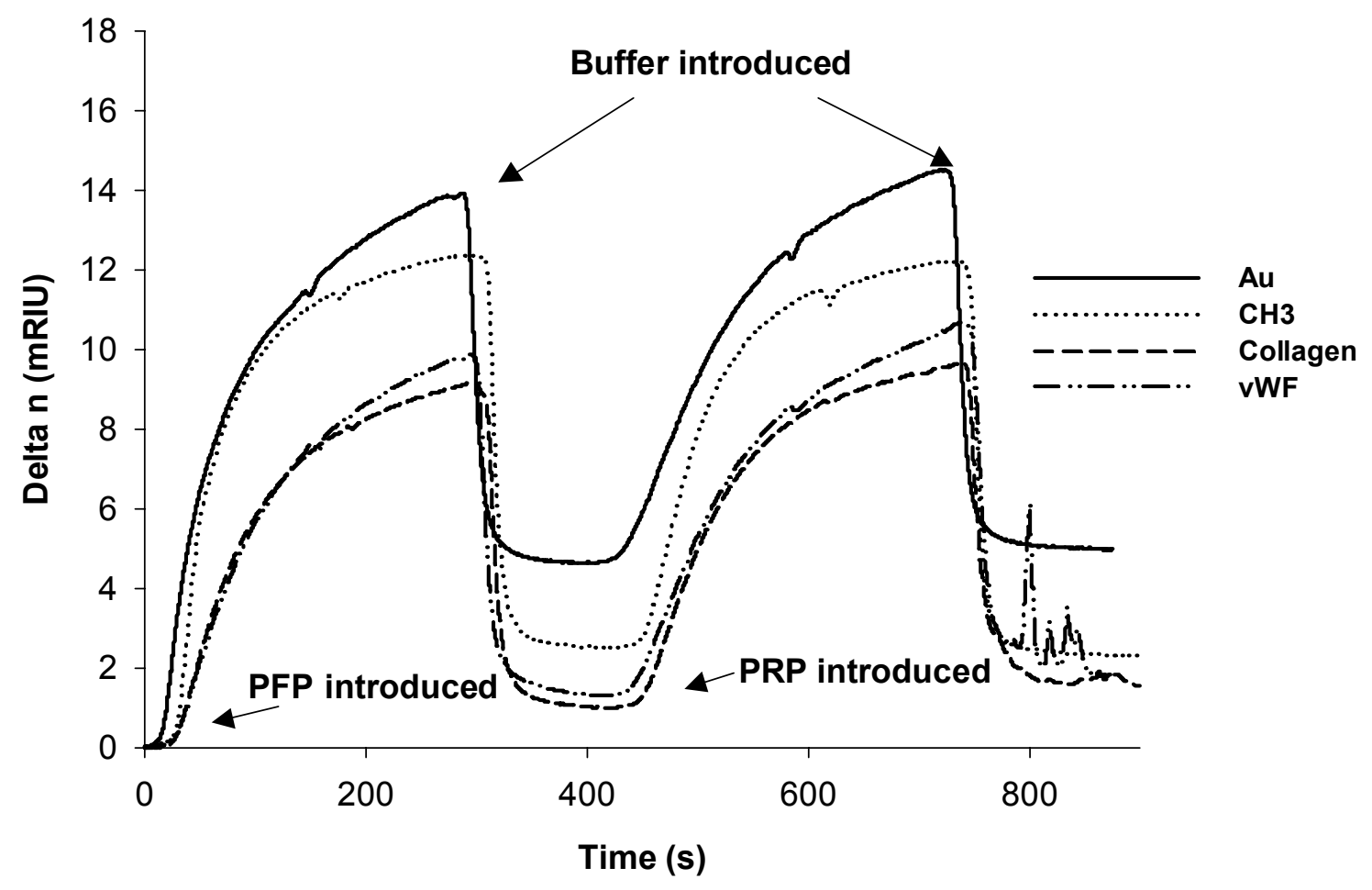

b)

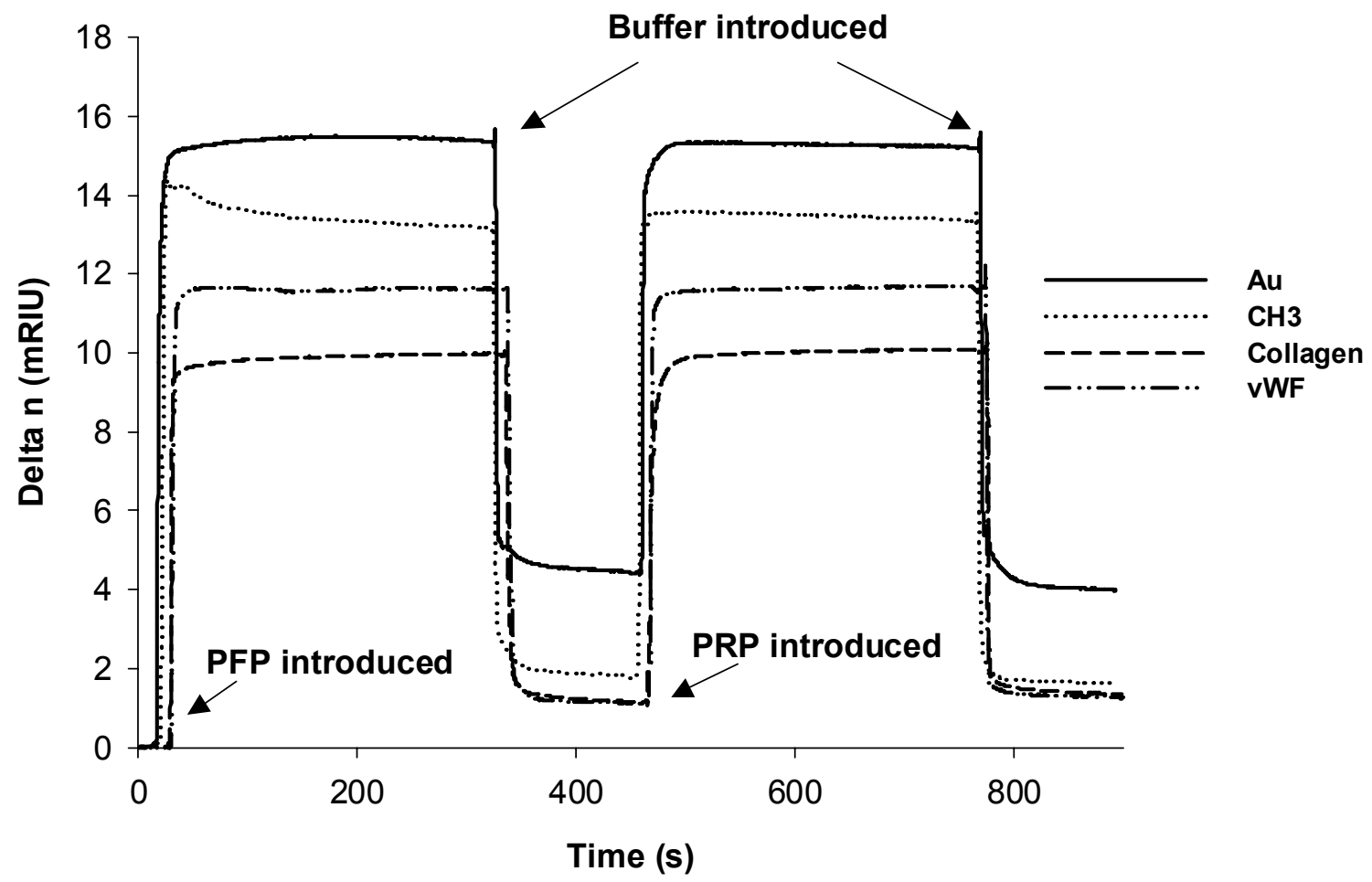


c)

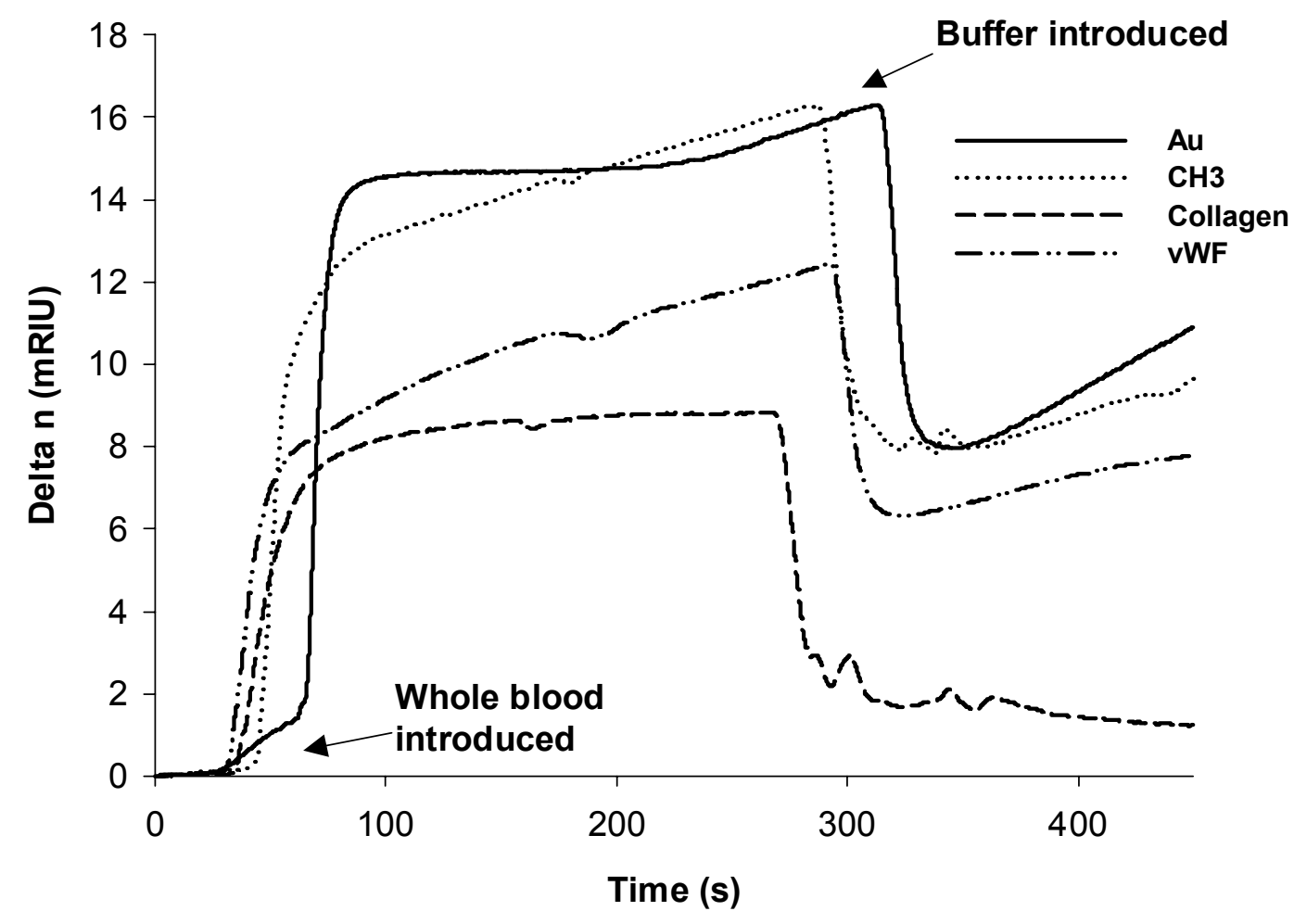

d)

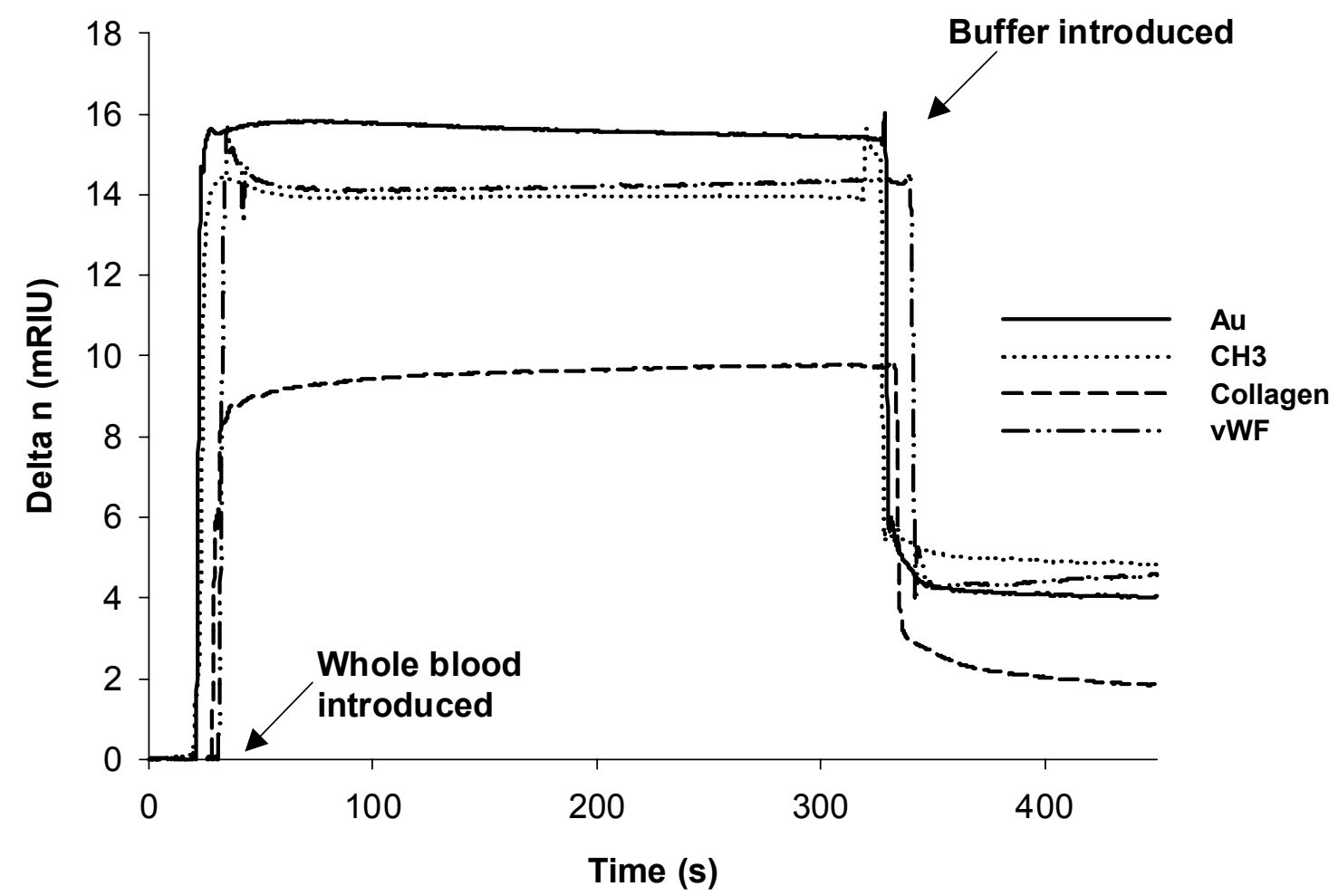


Supplement 2: Sensorgrams showing the SPR flow chamber signal when citrated recalcified PFP/PRP were passed through the flow chamber sequentially at a) venous (50 $\left.\mathrm{s}^{-1}\right)$ and $\left.\mathrm{b}\right)$ arterial $\left(1600 \mathrm{~s}^{-1}\right)$ shear rates, and recalcified citrated whole blood flown over the sensor surface at c) venous and d) arterial shear rates. Before and after the pulses of $\mathrm{PFP} / \mathrm{PRP} /$ whole blood there was a pulse with buffer to achieve stable conditions allowing estimation of how much proteins/cells that had adhered to the surface. The start for the different pulses are indicated with arrows in the figure. 